\title{
Migración interna y dinámicas laborales en la industria turística de la Riviera Maya, Quintana Roo, México
}

\author{
Internal Migration and Labor Dynamics in the Tourist Industry \\ of the Riviera Maya, Quintana Roo, Mexico \\ Anastacio Gustavo Fernández Rodríguez ${ }^{a}$ \\ gfernadez@uqroo.edu.mx
}

\author{
Mariana Figueroa de la Fuente ${ }^{b}$ \\ mfigueroa@uqroo.edu.mx \\ Ariel Ramón Medina Alonso ${ }^{c}$ \\ amedina@uqroo.edu.mx

\section{Mirna Yasmin Pacheco Cocom ${ }^{d}$ \\ mipcy@uqroo.edu.mx}

\begin{abstract}
a.b.c.d. Universidad de Quintana Roo, Departamento de Ciencias Administrativas, Unidad Académica de Playa del Carmen, México
\end{abstract}

Recibido: 22/09/2019 - Aceptado: 03/06/2020

\section{Resumen}

Los procesos migratorios internos o interestatales, cuya conceptualización implica el desplazamiento de personas dentro de un mismo Estado nacional, han sido estudiados como factores de diversas problemáticas del crecimiento económico a partir de la Segunda Guerra Mundial, este fenómeno social se ha acentuado en zonas costeras y ha contribuido al crecimiento

\section{(c) (1)(2)}


de polos turísticos como parte de la política turística desde los años setenta en México. A la vez, ha generado una importante demanda de empleo en el sector de los servicios, a la que ha respondido la fuerza laboral de otros Estados de la República mexicana y de las comunidades indígenas colindantes, y ha derivado implicaciones socioculturales, principalmente de identidad y aculturación. Por consiguiente, se personifica el migrante interno a través de su historia de movilidad, sus rutinas laborales en condiciones estacionarias con bajas remuneraciones en extenuantes jornadas de trabajo. En este contexto, la presente propuesta consta de un método cuantitativo-descriptivo, como parte de un proyecto de investigación de la Universidad de Quintana Roo, en Playa del Carmen y da paso la reflexión sobre la forma en cómo el crecimiento turístico genera un arrastre migratorio que ha ocasionado transformaciones culturales de usos y costumbres de los destinos de origen y, por otra parte, ha precarizado las condiciones laborales representadas por un modelo de desarrollo neoliberal de la industria de servicios en los destinos turísticos de la Riviera Maya, Quintana Roo, México.

Palabras clave: migración, derechos laborales, impactos del turismo, percepciones locales, Quintana Roo.

\begin{abstract}
Internal or inter-State migration processes, the conceptualization of which involves the displacement of persons within the same national State, have been studied as factors in various problems of economic growth since the Second World War, This social phenomenon has been accentuated in coastal areas and has contributed to the growth of tourist resorts as part of tourism policy since the 1970s in Mexico. This in turn has generated a significant demand for employment in the service sector, to which the labor force of other states of the Mexican Republic and the surrounding indigenous communities has responded, generating socio-cultural implications mainly of identity and acculturation. Consequently, the internal migrant is personified through his or her history of mobility, his or her work routines under stationary conditions with low pay in strenuous working days. In this context, the present proposal consists of a quantitative-descriptive method, as part of a research project
\end{abstract} Ariel Ramón Medina Alonso - Mirna Yasmin Pacheco Cocom

\title{
(ㄷ)(1) (2) (2)
}


of the University of Quintana Roo, in Playa del Carmen and gives way to the reflection on the way in which the tourist growth generates a migratory drag that has caused cultural transformations of uses and customs of the destinations of origin and, on the other hand, it has precarious working conditions represented by a neoliberal development model of the service industry in the tourist destinations of the Riviera Maya, Quintana Roo, Mexico.

Key Words: Migration, Labor rights, Tourism impacts, Local perceptions, Quintana Roo.

\section{INTRODUCCIÓN}

En una estrategia para diversificar los ingresos de México en los años setenta del siglo XX, el gobierno federal decidió apostar por el turismo como una alternativa económica del país, para llevar crecimiento económico a regiones aisladas e impulsar el "desarrollo" liderado por el Banco de México (BANXICO) y el Fondo de Promoción e Infraestructura Turística INFRATUR, hoy FONATUR (Vargas y Landero, 2014). En sintonía con la tendencia mundial, ya que en ese tiempo las cuencas turísticas del Mediterráneo, el Caribe y el Pacífico, especialmente Hawái, registraban altas tasas de crecimiento, por lo que la situación del mercado turístico internacional fue un factor decisivo en el nacimiento de los proyectos mexicanos. En ese sentido, Espinosa-Coria (2013) expone que los organismos internacionales como el "Banco Interamericano de Desarrollo (BID) facilitó los planes de las autoridades mexicanas, pues esta institución, al igual que el Banco Mundial, veía el turismo como una herramienta para promover el crecimiento y desarrollo de las naciones del llamado tercer mundo" (p. 158).

Por tanto, INFRATUR y BANXICO proyectaron a 25 años (1970-1995) el crecimiento turístico, con el objetivo de tercerizar la economía, incentivar la creación de empleos y la captación de divisas. En Quintana Roo, dichas estrategias originaron la ruta que va desde Puerto Juárez hasta Tulum, incluyendo Isla Mujeres y Cozumel, y con estas acciones el Estado mexicano hizo el cambio de una política económica turística (Magaña-Carrillo, 2008) a otra postura emprendedora, "empresa-Estado" (Boggio, 2014). Posteriormente, el Fondo Nacional de Fomento al Turismo (FONATUR, 2010), en la década de los ochenta, incentivó el desarrollo de megaproyectos turísticos, mediante planes maestros que incluyeron la venta masiva de

\section{(ㅇ)(1)(2) (2)}


tierras a inversionistas nacionales y extranjeros. De esta forma, en medio de devaluaciones y crisis económicas de este periodo, el Plan Nacional de Turismo 1980-1982 estableció, por primera vez, objetivos para los centros integralmente planeados (CIP). Para 1981, se comenzó con la construcción de la infraestructura de la segunda etapa de la zona turística de Cancún (Be-Ramírez et al., 2017). Con un crecimiento del $22 \%$, se construyeron, en promedio, 5.9 habitaciones por día, es decir, 10 mil 875 cuartos en el periodo (Vargas y Landero, 2014).

Debido al incremento en la ocupación hotelera en los años ochenta y noventa, Cancún se especializó en el turismo masivo, al edificar hoteles all inclusive y vuelos más asequibles. A partir de entonces, el crecimiento de la infraestructura hotelera y turística en la entidad ha sido exponencial en las últimas tres décadas, hasta consolidarse Cancún y Riviera Maya como los centros turísticos de sol y playa más importantes del país (Calderón y Orozco, 2008). En la actualidad, de acuerdo con las últimas cifras de indicadores turísticos publicadas por la Secretaría de Turismo del Estado de Quintana Roo (2017), la infraestructura hotelera en el Estado cuenta con 1067 hoteles y 100986 cuartos, de los cuales el $18 \%$ se encuentra en Cancún, el $40 \%$ en la Riviera Maya y el resto se distribuye en los otros municipios del Estado. Asimismo, en 2017, la derrama económica reportada fue de 2444.68 millones de dólares en Cancún y 1638.53 millones de dólares en Riviera Maya, de un total de \$4 592.41 millones de dólares generados por este sector en los principales puntos turísticos del Estado: Cozumel, Riviera Maya, Isla Mujeres, Chetumal y Mahahual. Es decir, el $53.2 \%$ se generó en Cancún y el $35.67 \%$ de la Riviera Maya, por lo que el turismo en la zona norte del Estado ha sido y sigue siendo el motor del crecimiento económico de Quintana Roo.

En este contexto, el libre mercado en América Latina ( $A L)$ propició la entrada de grupos turísticos internacionales (GTI) (Palafox, 2013), principalmente en zonas costeras del caribe mexicano, para implementar una infraestructura desmedida y brindar un crecimiento a zonas urbanas $t u$ ristizadas y mercantilizadas. Esta iniciativa comercial trajo consigo movilidades al Estado de forma vorágine y a su vez dicotómica. Por un lado, este desplazamiento ha servido como base de un crecimiento económico y habitacional y, por otro lado, lo antagónico de este crecimiento se refiere a los retrocesos y desigualdades (Gordillo y Plassot, 2017) laborales y socioculturales (Be, 2019; Sierra, 2007; Castellanos et al., 2006; García y

\section{()(1) (2)}


Gómez, 2018; Oehmichen, 2010). Ello, de forma indirecta, transgrede la cultura de las zonas de donde parten dichos sujetos y el papel que asumen al llegar a su nuevo destino en busca de mejores alternativas de trabajo, donde deben adaptarse al nuevo entorno turístico; además, la industria del turismo ha encontrado, a través de la normativa legal, la flexibilización del trabajo sobre los trabajadores, las nuevas generaciones que han quedado insertas en este medio y a lo que Be (2019) denomina "cuerpos dóciles", refiriéndose a estos colaboradores jóvenes del ocio.

Dadas estas premisas, la problemática se centra en entender: ¿Cómo se dan estas relaciones entre el patrón y trabajador en materia del derecho laboral de los trabajadores de la industria turística de la Riviera Maya, Quintana Roo? ¿Cuáles son las implicaciones culturales de los migrantes internos que salen de sus localidades en busca de mejores oportunidades laborales hacia los polos turísticos de la Riviera Maya? Por ello, el siguiente trabajo tiene como objetivo principal analizar el impacto que guarda la actividad turística sobre la historia migratoria de los trabajadores de la Riviera Maya, Quintana Roo y, a partir de ello, entender la relación patrón-trabajador, sus principales implicaciones laborales y socioculturales de esta movilidad interna hacia un destino turístico.

\section{Migración interna y condiciones laborales: "Flexibilización del trabajo"}

En este sentido, si bien la migración internacional no es el objetivo central de la presente investigación, resulta importante, para comprender su complejidad, conocer que cada año mil extranjeros migran al Estado, lo que, de acuerdo con el Instituto Nacional de Estadística y Geografía (INEGI)1, lo convierte en el quinto lugar nacional en la recepción de migración extranjera, con un $1.6 \%$ de su población nacida en otro país. Con todos los desafíos que esto representa para la administración del gobierno local, el número de extranjeros que viven en Quintana Roo incrementó a $27.52 \%$

1 El Instituto Nacional de Estadística y Geografía coordina el Sistema Nacional de Información Estadística y Geográfica en México (Gobierno de México, 2020). Y de conformidad con lo establecido por la Fracción I del artículo 59 de la Ley del Sistema Nacional de Información Estadística y Geográfica (LSNIEG, 2008, art.59) es el organismo que tiene la facultad exclusiva de realizar los censos nacionales; estos censos y en lo particular los poblacionales, y por recomendación de Naciones Unidas (1958) deben de realizarse cada 10 años, por lo cual datos estadísticos de censos poblacionales de 2010 en México son los últimos datos recientes y actualizados de forma oficial.

\section{(ㅇ)(1)(2) (2)}


al pasar de 18517 en 2010 a 23614 en 2015, lo que representa 5097 personas más que demandan servicios ${ }^{2}$.

En cuanto a la movilidad interna o movilización de personas de una entidad a otra dentro del mismo territorio, Quintana Roo reporta un $9.9 \%$ de personas que tienen menos de cinco años residiendo en la entidad, lo que representa uno de los porcentajes más altos de México, junto con el Estado de Baja California Sur (INEGI, 2018). Si bien es cierto que la migración en Quintana Roo es un fenómeno social importante en la entidad, se ha hecho evidente que hay contradicciones del desarrollo planeado de las principales ciudades, frente al crecimiento real generalmente desorganizado, consecuencia de la migración interesada en encontrar oportunidades en esta zona de México.

Ahora bien, la migración interna de forma rural-urbano se refiere al "migrante interno que se desplaza de una zona rural a una zona urbana" (OIM, 2006, p. 42) donde los individuos deciden su temporalidad y se desplazan dentro de su misma nación. Desde esta premisa, los centros integralmente planeados (CIP) se diseñaron como sello turístico, de captación de recursos para el Estado y, con ello, las movilidades entre regiones dieron paso a un proceso migratorio interno, en donde "presenta una nueva visión del ir y venir de bienes, personas y costumbres, y de su encuentro con el nuevo lugar y sus habitantes, tal como ocurre en la península de Yucatán" (Be, 2019, p. 10). En ese sentido, Sierra (2007) señala que en un comienzo el capital humano que provenía de zonas indígenas se empleaba en servicios turísticos hoteleros, construcción y cimentación de caminos. Esta migración interna hace alusión a que funciona en la forma en cómo el capital social se construye a través de alianzas y redes sociales que permiten la circulación del flujo migratorio entre paisanos (Be, 2015). Por esto, el movimiento de migrantes internos pasó de 3.48 a 6.4 millones, en tan sólo treinta años de 1995 a 2015 (CONAPO ${ }^{3}$ con base en Instituto Nacional de Estadística y Geografía (INEGI, 1995, 2000, 2010 y 2015), convirtiendo a Quintana Roo en líder en saldos netos migratorios (SNM).

2 Novedades de Quintana Roo, "Cada año, mil extranjeros hacen de Quintana Roo 'su hogar". sección "Media, Impresos", México, 27 de diciembre de 2015.

3 Organismo público de la Administración Pública Federal, creado en 1974 (LGP, 2018) con el objetivo de coordinar y dirigir las políticas nacionales de población.

\section{(2) (1) (2)}


Simultáneamente, esta migración interna representó experiencias colectivas e individuales a través del "sueño de progreso" (Re-Cruz, 2006, p. 150) como resultado de diversas problemáticas, donde el inmigrante transforma su realidad en la cual sus raíces son un poderoso activo de mercado. De esta forma, "los emigrantes mayas se familiarizan con la idea de que la cultura maya puede ser vendida, negociada, promovida y empaquetada" (Re-Cruz, 2006, p. 158); es decir, las personas que migran de lo rural a lo urbano conciben que su cultura puede ser comercializada y procesada como un producto turístico a granel, e incluso pueden ser empleados como imagen turística en la venta de artesanías, como una representación de un emblema cultural. Dichas acciones suelen crear conflictos, pues además contrastan las desigualdades de recursos al servicio del turista, con las condiciones precarias de sueldo, empleo y vivienda del inmigrante. Cabe mencionar que este sueño cancunense se construyó, primordialmente, por falta de identidad (Jiménez y Sosa, 2008) y la migración interna maya masculina en los años setenta y ochenta, hasta que finalmente la apertura comercial del Tratado de Libre Comercio de América del Norte (TLCAN) impulsó la inversión de capitales extranjeros que dan vida a los corredores de la Riviera Maya y Tulum (Sierra, 2007). ${ }^{4}$

Quintana Roo es un Estado puntero en movilidad tanto en la tendencia rural-urbana como urbana-urbana, a pesar de la propuesta de Pérez y Santos-Cerquera (2013), la cual versa en que el desplazamiento rural-urbano fue del siglo XX, mientras que para el siglo XXI los movimientos se presentan como urbano-urbano y urbanos-regionales. Desde el enfoque cuantitativo, los reportes de la Encuesta Nacional de la Dinámica Demográfica (ENADID, 2014) plantean la existencia de una migración interna de forma continua en relación con los años 2009 y 2014 del $3.4 \%$ y se identifican veinte entidades federativas que obtuvieron beneficios demográficos reflejados en crecimiento. Como principales resultados sociodemográficos se anotan las causas de la movilidad, entre las que destacan, en primer lugar, la reunión familiar (43.4\%); en segundo término, la búsqueda de oportunidades laborales (17\%), el cambio de empleo (10.3\%), la formación

4 Por tanto, es preciso indicar que las migraciones rurales a los polos turísticos del norte del Estado de Quintana Roo han sido una impronta de las investigaciones en la región, encarada desde diversos ángulos y abordajes analíticos (Brenner y Aguilar,2002; Calderón y Orozco, 2009; Calleja y González-Damián, 2016; Espinosa-Coria, 2013; Estrella, 2014; González y Macías, 2010; Jiménez y Zárraga, 2017; Méndez, 2013; Monterrubio et al., 2017; Moure, 2008; Palafox, 2013; Segrado et al., 2015; Sierra-Sosa y Ballesteros-Pérez, 2014, 2015; Tazim y Camargo, 2014; Torres y Momsen, 2005). 
educativa (7.5\%), la violencia e inseguridad (6.4\%), contraer matrimonio $(5.8 \%)$ y otras causas $(12.6 \%)$.

Por otro lado, las corrientes migratorias interestatales para los periodos 2005-2010, para el caso de Quintana Roo, pudieran considerarse peninsulares, donde la mayoría de los individuos emigran de los Estados de Tabasco, Chiapas, Campeche y Yucatán (Estimaciones del CONAPO con base en el INEGI, 2010). Es importante enmarcar que no sólo estos Estados contribuyen con las corrientes migratorias, sino que existe movimiento frecuente desde los Estados del Bajío, a pesar de que "desde los años cuarenta hasta mediados de los sesenta, la expansión de los sectores industriales y de servicios en las ciudades de México permitieron la incorporación de varios millones de migrantes rurales" (Arizpe, 1983, p. 31).

Por tanto, el fenómeno de la migración interna contiene un conjunto de máscaras y rostros diversos (Iglesias, 2011; Oehmichen, 2010) en función de las transformaciones económicas y de las movilidades. Por ello, muchas comunidades han perdido a casi todos sus jóvenes, principalmente varones, lo cual debilita su capacidad laboral, pero también tiene impactos en las normas y los valores culturales (Arnaiz y Dachary, 1992; Dachary, 1992; Hiernaux-Nicolás, 1999; Torres, 1994). En las ciudades receptoras, gran parte de la población económicamente activa se emplea en actividades del sector terciario, principalmente en el comercio al mayoreo y al menudeo, hospedaje y alimentos (INEGI, 2015). Sin embargo, ocupan, en la mayoría de los casos, puestos operativos mal remunerados de la industria hotelera y de servicios, como labores de limpieza, cocina, limpieza, jardinería, trabajo doméstico y de construcción, entre otros. Se crea, así, una división étnica del trabajo (Pi-Sunyer y Thomas, 1997; Torres y Momsen, 2014), un limitado acceso al mercado laboral, una precaria formación de trabajadores y la escasez de capital en estos espacios, lo cual todavía debe de ser resuelto (Brenner y Aguilar, 2002).

En ese orden de ideas, el capital humano que labora en Quintana Roo mantiene un promedio de su salario asociado a trabajadores asegurados en el Instituto Mexicano del Seguro Social es de \$7 584.7 pesos mexicanos, $25.8 \%$ más bajo que la media nacional de $\$ 10222.3$ pesos mexicanos (Subsecretaría de Empleo y Productividad Laboral, 2018). Además, se redujo la capacidad de compra del ingreso laboral en $1.3 \%$, lo cual contrasta con el costo que representa vivir en ciudades como Cancún,

\section{(1) (1) (2)}


catalogada como la segunda ciudad más cara para vivir en México, después de los Cabos y seguida por Monterrey (CNN, 2015).

Todo esto forma parte de una realidad que discrepa de lo expresado en los artículos 5 y 123 de la Constitución Política de los Estados Unidos Mexicanos (CPEUM), que garantizan para todos los mexicanos la libertad y el derecho humano y fundamental al trabajo, respectivamente. La Constitución, a través de este artículo, prohíbe la celebración de contratos, pactos o convenios que vayan en contra de las libertades de las personas, reconocidas por la propia Constitución General. Éstos tampoco podrán admitir, la autoproscripción o destierro de la persona, o que ésta renuncie temporal o permanentemente a ejercer determinada profesión, industria o comercio. La proscripción y el destierro se refieren a obligarse a salir, sin posibilidad de regreso al territorio de la patria. Finalmente, el artículo 5 constitucional, señala:

[El contrato de trabajo] no podrá extenderse, en ningún caso, a la renuncia, pérdida o menoscabo de cualquiera de los derechos políticos o civiles.

Es decir, en términos generales, el ejercicio de la libertad laboral a la que hace referencia y garantiza la Carta Magna nunca podrá ir en detrimento de las demás libertades y derechos consagrados por la propia Ley Fundamental. Por otra parte, la CPEUM, promulgada el 5 de febrero de 1917, reconoce diversas "garantías sociales" en idéntico rango que, a las garantías constitucionales, las mismas que hoy son denominadas "derechos fundamentales". Fijan, entre otras garantías, la jornada laboral de 8 horas, un día de descanso semanal, salario mínimo general, pago de horas extras a la jornada ordinaria, derecho a la vivienda digna para el trabajador y su familia, riesgos de trabajo, entre otras.

De igual forma, la Ley Federal del Trabajo establece una serie de derechos irrenunciables de los trabajadores como: vacaciones, prima vacacional, días de descanso obligatorios, aguinaldo, prima de antigüedad, participación de los trabajadores en el reparto de utilidades, alimentación y habitación, estímulos, etc. En suma, es amplio el abanico de derechos establecidos en la Constitución y en la Ley para la protección de los trabajadores, el reto, sin duda, recae en su respeto y aplicación, y que se ha catalogado como precarización o flexibilización laboral (Bosch, et al., 2004; Cárdenas, 
2015; Castel, 2010; Palafox y Rubí, 2015). En ese sentido, hablar de migración interna y condiciones laborales en el destino turístico de la Riviera Maya brinda una aproximación sobre el espacio en donde se desenvuelve el trabajador migrante y, de esta manera, se comprende su simultaneidad e implicaciones sociales (Cárdenas, 2014) junto al fenómeno del turismo (Be-Ramírez et al., 2017) y la instrumentación para el empresarial desarrollo (Varela et al., 2017) como una de las políticas del sistema neoliberal.

\section{MÉTODO}

Para el presente trabajo se utilizó un enfoque metodológico cuantitativo-descriptivo, que partió de la revisión bibliográfica $y$, con base en la propuesta del Instituto para la Seguridad y la Democracia A.C., se diseñó un cuestionario modificado y adaptado con información a Quintana Roo, las relaciones entre el patrón y trabajador en materia del derecho laboral de los trabajadores de la industria turística de la Riviera Maya, Quintana Roo, así como las implicaciones culturales de los migrantes internos en la zona. El tipo de muestreo utilizado fue no probabilístico para aquellos participantes que estuvieran económicamente activos en la Riviera Maya y fueran migrantes internos. Por lo tanto, se trabajó una muestra finita de la población económicamente activa (PEA), que reporta la Subsecretaría de Empleo y Productividad Laboral en el apartado de Quintana Roo y, establece un total de 890199 la PEA y un índice de 864429 trabajadores ocupados, mientras que 25700 con la etiqueta de desocupados. Con estos datos se calculó el número de cuestionarios para aplicar a través de la siguiente formula:

$$
n=\frac{N}{1+\frac{e^{2}(N-1)}{z^{2} p q}}
$$

Sustituyendo sus valores la $\mathrm{N}=864429$, el error estimado se trabajó a un $5 \%$, mientras que el valor del nivel de confianza fue del $95 \%$ y una probabilidad de éxito (p) y fracaso (q) del $50 \%$ cada uno. El resultado fue de 383 cuestionarios por realizar. Posteriormente, se llevó a cabo la recolección de información en tres fases: la primera se realizó en mayo de 2018, la segunda en noviembre de 2018 y finalmente en febrero de 2019, en donde se recopilaron 471 participaciones, y después de una depuración

\section{(c) (1) (2) (2)}


por información incompleta, se quedaron para analizar 445 cuestionarios finales. A continuación, se presentan de manera descriptiva los hallazgos.

\section{ANÁLISIS Y DISCUSIÓN DE RESULTADOS}

Los resultados obtenidos desde el perfil sociodemográfico de los participantes se componen de la siguiente manera: un $54.6 \%$ de la muestra representa al sexo masculino y un $45.4 \%$ son mujeres. Es importante resaltar que el $20.2 \%$ del tamaño muestral son personas provenientes de Yucatán, el $4.3 \%$ del Estado de Campeche, el $12.6 \%$ de Chiapas, el 8.5 $\%$ del Tabasco, el $10.6 \%$ del Estado de Veracruz, $6.7 \%$ de la CDMX, el $3.1 \%$ del Estado de Puebla, entre los más representativos y con similitud, de acuerdo con las estimaciones del CONAPO e INEGI (2010). Además, el movimiento migratorio interestatal hacia la Riviera Maya representa el $15.9 \%$ proveniente, principalmente, de los municipios de Benito Juárez, Chetumal, Felipe Carrillo Puerto, Bacalar, José María Morelos y las comunidades de Chunhuhub, Manuel Antonio Hay, Melchor, Naranjal, Saban, San José II, Tihosuco y X-pichil. En ese contexto, Torres y Momsen (2014) plantean que esta salida del lugar de origen por parte de los emigrantes ha fortalecido la industria del turismo, en puestos mayormente operativos $y$, en ese mismo sentido, se ven transformados sus valores y adoptan otro rol en el sitio donde deciden residir (Arnaiz y Dachary, 1992; Etzo, Massida y Piras, 2014; Hiernaux-Nicolás, 1999; Iglesias, 2011).

Los participantes afirman saber leer y escribir en un $94.6 \%$ y su nivel educativo se concentra entre secundaria y licenciatura (primaria $9.2 \%$; secundaria 21. $8 \%$; preparatoria $36.8 \%$; licenciatura $30.3 \%$ ). La edad promedio oscila entre los 20 y 29 años (51.4\%) y de 30 a 39 años representa un $17.7 \%$, por lo que se puede notar una población joven en busca de oportunidades laborales, con una preparación educativa básica y con el anhelo de mejorar su nivel de vida. El $54.8 \%$ reportó que su ingreso quincenal es de más de 4000 pesos mexicanos, $52.8 \%$ son solteros, el $29.4 \%$ son casados y el $17.8 \%$ está comprometido por unión libre.

El principal motivo de migración reportado fue la superación económica en un $\quad 64.7 \%$, reunión familiar en un $13.9 \%$ (Etzo, Massida y Piras, 2014 ), inseguridad en su lugar de origen en un $5.2 \%$, amenazas y persecución en un $0.4 \%$, el $13.3 \%$, otros sin especificar y un $2.5 \%$ se abstuvo de responder esta pregunta. Este resultado concuerda con lo expuesto

\section{(ㅇ)(1)(2) (2)}


por la ENADID (2014) y la búsqueda del progreso con Re-Cruz (2006) y Sierra (2007). Enfocan como principal destino la Riviera Maya en un $84 \%$, mientras que Cancún era visualizado como destino final para un $9.9 \%$ y el $6.1 \%$ no contestó. Este movimiento migratorio, de acuerdo con los datos proporcionados por los participantes, comienza a ser más representativo desde 1997 y de ahí ha ido en aumento hasta 2019, lo cual concuerda con la apertura del TLCAN, con la expansión de los mercados y la entrada de los GTI (Palafox, 2013).

Los resultados referentes a las veces que ha emigrado el participante señalan que un $67.4 \%$ lo ha realizado por primera vez, el $19.4 \%$ lo ha llevado a cabo en dos ocasiones, un $7.9 \%$ por tercera vez y un $5 \%$ más de 4 veces. Esto parece indicar que la mayoría ha encontrado estabilidad en su primera salida de su lugar de origen y se ha asentado en el Estado de Quintana Roo. En estos flujos migratorios, regularmente, los que emigran viajan acompañados (56.6\%), en otras ocasiones de forma autónoma (40.4\%) y un $2.9 \%$ se contuvo de responder. Quienes viajan acompañados suelen hacerlo con familiares (44\%), con un amigo (11\%) y con un conocido (3.4\%); no emitieron respuesta alguna en un $\%$. Finalmente, el medio de transporte que utilizaron los participantes para llegar a Quintana Roo fue terrestre (71\%), el $20.2 \%$ vías aéreas y un 7.8 $\%$ no contestó.

Respecto al momento de regresar a su lugar de origen, la frecuencia reportada fue de una vez al año en un $36.2 \%$ de los participantes. Las razones por las que no visitan a sus familiares fueron por falta de vacaciones o días feriados (47.6\%), así como el elevado costo de desplazamiento a su lugar de origen (24.3\%). Este resultado señala que, a pesar de haber encontrado estabilidad laboral, los costos que representa vivir en la entidad son altos; por otro lado, se tendría que verificar a qué tipo de contratos están sujetos los trabajadores, de acuerdo con lo que expresa la Ley Federal del Trabajo en su capítulo II.

Por otro lado, las formas de comunicación que utilizan los participantes con sus familiares son por vía telefónica en su mayoría (83.1\%), vía electrónica en un $3.8 \%$ y por el correo postal un $1.1 \%$. Como gratificación suelen enviar regularmente dinero su lugar de origen en un $45.4 \%$, mientras que un $47.9 \%$ no lo realiza. Este apego a la familia en ocasiones se ve en el envío de presentes a su lugar de origen en un $60.3 \%$. Respecto a las

\section{(c) (1) (2) (2)}


oportunidades de empleo en la Riviera Maya, el $83.6 \%$ de los participantes afirma contar con un trabajo remunerado, es decir, con base salarial, en jornadas que oscilan entre 10 y 72 horas. También se encontró que el $68.3 \%$ de los colaboradores labora los sábados y un $43.6 \%$ lo hace los domingos, ya que generalmente son los días de mayor actividad en el ramo turístico.

Figura 1.

Paradero de colaboradores de la industria turística "Misión del Carmen", Solidaridad

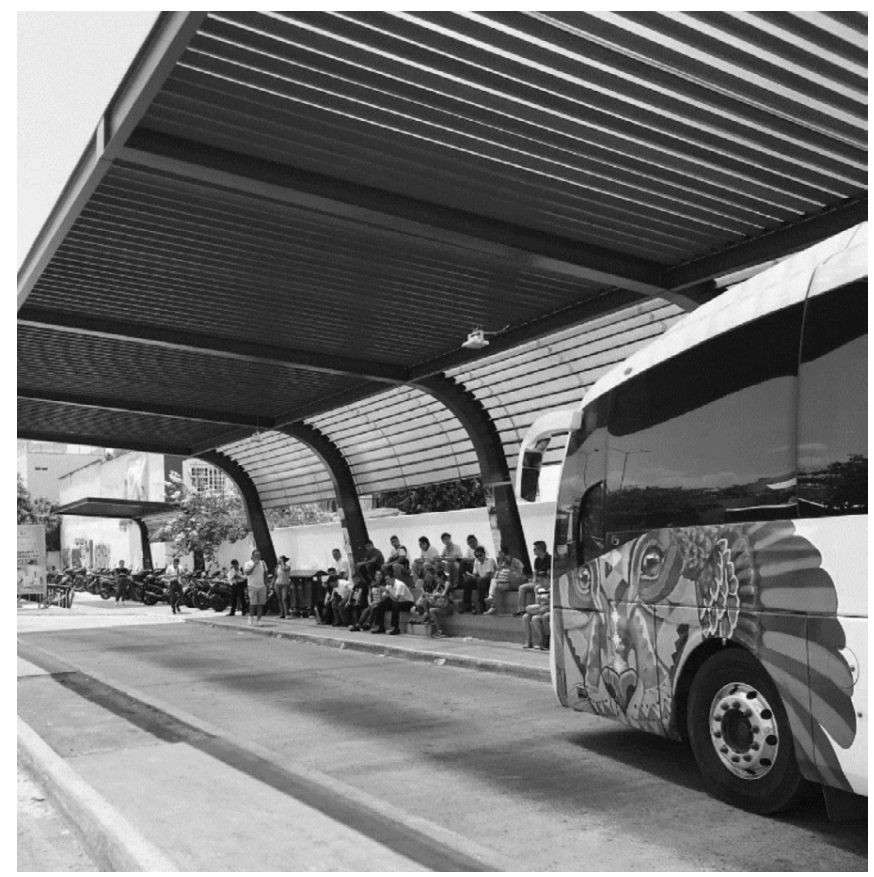

Nota: Archivo personal (2019).

De los participantes encuestados con hijos, el $23.6 \%$ reporta que estos se encuentran bajo el cuidado de sus familiares, el $9.9 \%$ se ocupa de forma personal de ellos, el $1.8 \%$ los dejan con amigos, el $6.9 \%$ pagan por servicios de guardería o niñeras. Dentro de los menores que se encuentran cursando la educación básica, la mayoría pertenece a escuelas públicas $(27.4 \%)$, mientras que el $5.6 \%$ estudian en escuelas privadas.

\section{(2)(1)(2)}


Este resultado denota que los ingresos que se perciben en la familia son destinados a otros gastos en función del salario. En ese mismo tenor, el promedio del salario de los trabajadores es de $\$ 7584.7$ o su equivalente \$341.30 dólares estadounidenses (Subsecretaría de Empleo y Productividad Laboral, 2018). No obstante, algunos trabajadores suelen duplicar este salario base, por una práctica basada en el modelo americanizado de propinas, beneficio del que gozan.

Finalmente, un $22.2 \%$ habla una lengua indígena. Entre las principales lenguas indígenas que se hablan en Quintana Roo se encuentra la lengua maya con un $19.3 \%$, tzotzil $(1.3 \%)$, Chol $(0.9 \%)$ y tzeltal $(0.8 \%)$. Este dato resulta relevante de considerar, pues, usualmente, al migrar pierden su lengua materna (Re-Cruz, 2006). El uso de las lenguas vernaculares se proyecta como un atractivo más para el turismo en los parques temáticos que tienden a la disneyzación de la cultura y la macdonalización de la gastronomía (Córdoba, 2009) en un espacio de culturas híbridas (Canclini, 1997).

\section{CONCLUSIONES}

En conclusión, la migración interna, en términos culturales, económicos e identitarios, ha conllevado a diversos procesos de pérdida; pero también ha propiciado reconfiguraciones culturales. Los resultados obtenidos dejan ver que estos procesos migratorios internos en Quintana Roo se realizan en busca de mejores condiciones de vida. En este contexto, si bien es cierto que los trabajadores, migrantes internos, consideran que es positivo contar con diversidad de fuentes de empleo, otra lectura permite advertir que las personas no logran mejorar su nivel de vida, ni en lo económico, ni en el tipo de educación o cuidado que pueden dar a sus hijos. Estas cuestiones obstaculizan su regreso, ya sea de visita o definitivo al lugar de origen, por falta de vacaciones o por salarios mal remunerados, además de las inconstitucionales e ilegales relaciones contractuales ejercidas entre patrón-trabajador.

El panorama del crecimiento de Quintana Roo ha hecho que la estructura social y laboral sea compleja; los grupos de poder representados por el Estado y las franquicias internacionales, nacionales y extranjeras se vuelven actores clave en la acumulación del capital y riqueza. En ese sentido, la industria del turismo quintanarroense se ha visto envuelta en el aprovechamiento empresarial hacia la explotación de mano de obra no calificada,

\section{() (1)(2)}


que toma como ventaja las movilidades de sectores vulnerables, principalmente rurales, $y$, de esta forma, han convencido de que los bienes culturales y comunes pueden ser aprovechados como oferta al turismo por parte de las comunidades anfitrionas, al escenificar la cultura. Dichas movilidades han suplantado la cultura, la lengua, la vestimenta, debido a las oleadas migratorias que día tras día flotan en una región. En este caso, para la Riviera Maya, la población yucateca se ha despojado de sus vestimentas originales para favorecer e insertarse en la lucha empresarial de corte trasnacional.

Estas ideas ratifican la importancia de la elaboración de estudios comparativos, detallados, basados en el trabajo de campo y que den cuenta de los impactos específicos sociales, económicos y medio ambientales de las distintas formas de turismo. Además, de la realización de investigación aplicada, que pueda ofrecer información de cómo incrementar los beneficios del turismo a nivel local y regional, como lo han señalado diversos autores entre los que destacan Brenner y Aguilar (2002).

Esto ofrecerá información valiosa a las instancias gubernamentales y a la iniciativa privada para la toma de decisiones, además de permitir abrir el debate de las problemáticas existentes, para poder hacer una reformulación del modelo de desarrollo turístico enfocado hacia la sostenibilidad y el respeto a los derechos humanos en Quintana Roo. De igual manera, en una línea futura investigación se puede profundizar sobre los oficios que ejercen los migrantes o sus antecesores, y conocer la modificación de sus actividades económicas.

Agradecimientos: A todos los estudiantes de la carrera de Administración Hotelera de la Universidad de Quintana Roo, Unidad Académica Playa del Carmen, que contribuyeron en este proyecto de investigación.

\section{REFERENCIAS}

Arizpe, L. (1983). El éxodo rural en México y su relación con la migración a Estados Unidos. Estudios Sociológicos, 9-33.

Arnaiz, S., y Dachary, A. (1992). Cancun: El enclave turístico y sus costos. In Caribbean Studies Association XVII Annual Conference, Los Impactos Sociales y Económicos del Turismo, Grenada, West Indies.

\section{(ㅇ)(1) (2) (2)}


Banco de México. (1973). Restructuración del proyecto Cancún. Fondo Antonio Enríquez Savignac (Carpeta INFRATUR). Biblioteca de la Universidad del Caribe, Cancún.

Be, R.P.A. (2015). Migrantes yucatecos, itinerarios transnacionales y aprendizajes, la experiencia desde un escenario turístico. Cuicuilco, $64,63-87$.

Be, R.P.A. (2019). Segunda generación de migrantes yucatecos en México: Los "braceros del ocio" como mercancía inmaterial de un paraíso turístico. Revista latinoamericana de antropología del trabajo, 5, $1-25$.

Be-Ramírez, P. A., Lozano, S., Gabriel, E., y González Neri, A. (2017). Prácticas socioculturales, turismo e identidad entre los hijos de migrantes yucatecos en Cancún. LiminaR, 15(2), 155-168.

Boggio, J. (2014). Cancún, una empresa de Estado. En J. Arriaga (Coord.), XIX Congreso Internacional de la Asociación Mexicana de Estudios del Caribe (AMEC). Conferencia impartida en la Universidad de Quintana Roo, Chetumal, QR.

Bosch, J. L., Suárez, S., \& Olivares, G. (2004). La importancia de la generación de empleo como dinamizadora del desarrollo local en un centro turístico. Caso: San Carlos de Bariloche. Aportes y transferencias, 8(2), 25-44.

Brenner, L., y Aguilar, A.G. (2002). Turismo de lujo y desarrollo económico regional en México. El geógrafo profesional, 54(4), 500-520.

Calderón Maya, J. y Orozco Hernández, M. (2009). Planeación y modelo urbano: El caso de Cancún, Quintana Roo. Quivera, 11(2), 18-34. http://www.redalyc.org/pdf/401/40113786002.pdf

Calderón, J. y Orozco, M. (2009). Planeación y modelo urbano: El caso de Cancún, Quintana Roo. Quivera, 11(2), 18-34.

Calleja Sordo, C., y González Damián, A. (2016). Mercantilización de la cultura en aras de ofrecer una experiencia turística estandarizada. 
Reflexiones desde el caso de Cozumel, México. Revista Iberoamericana De Turismo, 682-95. 10.2436/20.8070.01.15

Canclini, N. (1997). El malestar en los estudios culturales. En Fractal. Revista Trimestral [on line], 2(6), 45-60. http://www.fractal.com.mx/ F6cancli.html

Cárdenas, G. E. P. (2014). La precariedad laboral de los migrantes chiapanecos en la construcción del Mayan Palace de Nuevo Vallarta. Aposta, 65, 111-130.

Cárdenas, G. E. P. (2014). Migración interna e indígena en México. Intersticios sociales, 7, 1-28.

Castel, Robert. (2010). El ascenso de las incertidumbres. Trabajo, protecciones, estatuto del individuo. FCE.

Castellanos Ortega, M. L. y Pedreño Cánovas, A. (2006). Los nuevos braceros del ocio. Sonrisas, cuerpos flexibles e identidad de empresa en el sector turístico. Miño y Dávila Editores.

CNN. (2015). Las 3 ciudades más caras para vivir en México. https://expansion.mx/economia/2015/01/27/ las-5-ciudades-mas-caras-y-baratas-para-vivir-en-mexico

Consejo Nacional de Evaluación de la Política de Desarrollo Social. (2017). Índice de la tendencia laboral de la pobreza en Quintana Roo. CONEVAL. https://www.coneval.org.mx/coordinacion/entidades/QuintanaRoo/Paginas/itlp.aspx

Córdoba, O. J. (2009). Turismo, desarrollo y disneyzación: ¿Una cuestión de recursos o de ingenio? Investigaciones geográficas, 70, 33-54.

Dachary, A. (1992) Quintana Roo: Los retos del fin de siglo. ¿Hacia un desarrollo desigual? In A. Dachary, D. Navarro López and S.M. Arnaiz (Eds.), Quintana Roo: Los retos del fin de siglo (pp. 9-29). Centro de Investigaciones de Quintana Roo.

ENADID. (2014). Encuesta nacional de la dinámica demográfica. Principales resultados. http://internet.contenidos.inegi.org.mx/contenidos/ 
productos/prod_serv/contenidos/espanol/bvinegi/productos/nueva_ estruc/promo/resultados_enadid14.pdf

Espinosa-Coria, H. (2013). El origen del proyecto turístico Cancún, México: Una valoración de sus objetivos iniciales a 42 años de su nacimiento. LiminaR, 11(1), 154-167.

Estrella, C. E. (2014). Finanzas públicas municipales y turismo. Revista Electrónica CECIET, 3(7), 31-43.

Etzo, I., Massida, C. y Piras, R. (2014). Migration and outbound tourism: evidence from Italy. Annals of Tourism Research, 48, 235-249.

Fondo Nacional de Fomento al Turismo. (2010). FONATUR 35 años. Una historia narrada por sus fundadores y protagonistas. FONATUR.

García, T. A. C. y Gómez, G. A. (2018). Migración interna en Nayarit: Crecimiento poblacional en Bahía de Banderas. Revista Educateconciencia, 18(19), 20-30.

Gobierno de México. (2018). Ley del Sistema Nacional de Información Estadística y Geográfica. Última reforma publicada DOF (25-06-2018). http://www.diputados.gob.mx/LeyesBiblio/pdf/LSNIEG_250618.pdf

Gobierno de México. (2018). Ley General de Población. Última reforma publicada DOF (12-07-2018). https://observatoriocensal.files.wordpress.com/2017/08/series_m27en.pdf

Gobierno de México. (2019). Ley Federal del Trabajo. Última reforma publicada DOF (02-07-2019). http://www.diputados.gob.mx/LeyesBiblio/ pdf/125_020719.pdf

Gobierno de México. (2020). Constitución Política de los Estados Unidos Mexicanos. Última reforma DOF (08-05-2020). http://www.diputados. gob.mx/LeyesBiblio/pdf_mov/Constitucion_Politica.pdf

Gobierno de México. (2020). Creación del INEGI. https://www.gob.mx/ siap/articulos/creacion-del-inegi

94 Anastacio Gustavo Fernández Rodríguez - Mariana Figueroa de la Fuente Ariel Ramón Medina Alonso - Mirna Yasmin Pacheco Cocom

\section{()(1) (2) (2)}


González D., y Macías, A. (2010). Tres décadas como anfitriona: Génesis, expansión y crisis de la sociedad quintanarroense. Revista de economía, sociedad, turismo y medio ambiente: RESTMA, 11, 193-221.

Gordillo, G., y Plassot, T. (2017). Migraciones internas: Un análisis espacio-temporal del periodo 1970-2015. Economía UNAM, 14(40), 67-100.

Hiernaux-Nicolás, D. (1999) Cancún bliss. In D. Judd and S. Fainstein (Eds.), The Tourist City (pp. 124-39). Yale University Press.

Iglesias, E. L. (2011). Las nuevas migraciones yucatenses: territorios y remesas. Migración y Desarrollo, 9(17), 69-90.

Instituto Nacional de Estadística y Geografía (INEGI). (2015a). Principales resultados de la Encuesta intercensal 2015. INEGI.

Instituto Nacional de Estadística y Geografía (INEGI). (2015b). Actividades económicas. http://cuentame.inegi.org.mx/monografias/informacion/ qroo/economia/

Instituto Nacional de Estadística y Geografía (INEGI). (2018). INEGI Encuesta Nacional de la Dinámica Demográfica 2018. https://www. inegi.org.mx/temas/migracion/

Jiménez M. A. de J. y Sosa, P. (2008). Cocktail Cancún: reflexiones sobre los impactos sociales del turismo en la comunidad local. En Maribel Osorio G. y Marcelino Castillo N. (Coords.), Entorno del turismo. Perspectivas (pp. 63-109) Universidad Autónoma del Estado de México.

Jiménez. M. y Zárraga, L. (2017). Impactos socioculturales del emprendedor en el polo de desarrollo turístico del Estado de Quintana Roo, desde un enfoque territorial. Regions and Cohesion, 7(2), 70-89.

Magaña-Carrillo, I. (2009). La política turística en México desde el modelo de calidad total: un reto de competitividad. Economía, sociedad y territorio, 9(30), 515-544. 
Méndez, E. (2013). De dinámicas migratorias a biografías ingrávidas en la Riviera Maya. En Anuario digital CEMI. Centro de estudios de migraciones internacionales Universidad de la Habana.

Monterrubio, C., Osorio, M., \& Benítez, J. (2017). Comparing enclave tourism's socioeconomic impacts: A dependency theory approach to three state-planned resorts in Mexico. Journal of Destination Marketing \& Management.

Moure, M. (2008). Cultura maya en el contexto de la globalización: Turismo internacional y migración [Tesis de licenciatura]. Universidad de Quintana Roo. http://risisbi.uqroo.mx/handle/20.500.12249/459

Oehmichen, C. (2010), Cancún: la polarización social como paradigma en un México Resort. Alteridades, 20(40), 23-34.

Organización Internacional para las Migraciones (OIM). (2006). Glosario sobre migración. OIM.

Palafox, A. M. (2013). El turismo como eje de acumulación. Nómadas. Critical Journal of Social and Juridical Sciences, Especial: América Latina, 161-174. https://doi.org/10.5209/rev_NOMA.2013.42347

Pérez, C. y Santos-Cerquera, C. (2013). Tendencias recientes de la migración interna en México. Papeles de población, 19(76), 53-88.

Pi-Sunyer, O. and Brooke Thomas, R. (1997) Tourism, environmentalism, and cultural survival in Quintana Roo. In B.R. Johnson (Ed.), Life and Death Matters: Human Rights and the Environment at the End of the Millennium (pp. 187-212). AltaMira.

Re-Cruz. (2006). Turismo y migración entre los mayas de Yucatán. Las nuevas milpas de Chan Kom. Revitas Española de Antropología americana, 36(1), 149-162.

Rubí-González, F. y Palafox-Muñoz, A. (2015). El turismo como catalizador de la pobreza. Trabajo turístico y precariedad en Cozumel, México. Alba Sud. 
Secretaría de Turismo del Estado de Quintana Roo. (2017). Indicadores turísticos 2017. SEDETUR. http://caribemexicano.travel/ARCHIVOS/ indicadores/Indicadores\%20Tur\%20-\%20Junio\%202017.pdf

Segrado Pavón, R. G., Arroyo Arcos, L., Cruz Jiménez, G., Serrano Barquín, R. C. y Juan Pérez, J. I. (2015). Estrategias de competitividad turística en las áreas naturales protegidas de Quintana Roo, México. Investigaciones Turísticas, 9, 1-23.

Sierra, S. L. A. (2007). Migración y trabajo en el Caribe mexicano. Entre dos mundos, las familias mayas y el turismo masivo. En Urbanización y turismo (pp. 25-38). Universidad de Quintana Roo.

Sierra-Sosa, L. y Ballesteros-Pérez, X. (2014). Rutas cotidianas y recorridos migratorios en dos comunidades de la Zona Maya de Quintana Roo. Revista Mundi Migratios, 2(2), 127-142. http://biblioteca.clacso. edu.ar/Cuba/cemi-uh/20150911010617/4-53-100-1-SM.pdf

Sierra-Sosa, L. y Ballesteros-Pérez, X. (2015). Pueblos de apoyo en contextos turísticos. Akumal, enclave turístico de la Riviera Maya de Quintana Roo. Temas antropológicos: Revista científica de investigaciones regionales, 37(2), 121-139.

Subsecretaría de Empleo y Productividad Laboral. (2018). Información laboral febrero 2018. STPS. http://www.stps.gob.mx/gobmx/estadisticas/pdf/perfiles/perfil\%20quintana\%20roo.pdf

Tazim, J. y Camargo, B. (2014) Sustainable tourism, justice, and an ethic of care: toward the Just Destination. Journal of Sustainable Tourism, 22(1), 11-30. 10.1080/09669582.2013.786084

Torres Maldonado, E. J. (1994). Desarrollo turístico, TLC y cambio social en la frontera sur de México: El caso de Quintana Roo. Anuario de Estudios Urbanos, 1, 215-57.

Torres, R. M., y Momsen, J. D. (2005). Gringolandia: The construction of a new tourist space in Mexico. Annals of the Association of American Geographers, 95(2), 314-335. 10.1111/j.1467-8306.2005.00462.x 
Torres, R. y Momsen, J. (2005) Planned Tourism Development in Quintana Roo, Mexico: Engine for Regional Development or Prescription for Inequitable Growth? Current Issues in Tourism, 8(4), 259-285. $10.1080 / 13683500508668218$

Varela Llamas, R., Ocegueda Hernández, J. M., y Castillo Ponce, R. A. (2017). Migración interna en México y causas de su movilidad. Perfiles latinoamericanos, 25(49), 141-167.

Vargas, M. y Landero, R. (2014). Turismo y desarrollo en Quintana Roo. Una visión legislativa en su 40 aniversario ( $\left.2^{\mathrm{da}} \mathrm{ed}\right)$. Editorial Progreso. Ariel Ramón Medina Alonso - Mirna Yasmin Pacheco Cocom

\section{(ㄷ)(1) (2) (2)}

